\title{
Choosing the Smoothing Parameters within a Multiple-Pass Barnes Objective Analysis Scheme: A Cautionary Note
}

\author{
PHILLIP L. SPENCER*,+ \\ Cooperative Institute for Mesoscale Meteorological Studies, University of Oklahoma, Norman, Oklahoma \\ Mark A. Askelson \\ Department of Atmospheric Sciences, University of North Dakota, Grand Forks, North Dakota \\ Charles A. Doswell III \\ Cooperative Institute for Mesoscale Meteorological Studies, University of Oklahoma, Norman, Oklahoma
}

(Manuscript received 17 March 2006, in final form 11 July 2006)

\begin{abstract}
Various combinations of smoothing parameters within a two-pass Barnes objective analysis scheme are applied to analytic observations obtained by regular and irregular sampling of a one-dimensional sinusoidal analytic wave to obtain gridded fields. Each of these various combinations of smoothing parameters would produce equivalent analyses if the observations were continuous and infinite (unbounded). The authors demonstrate that owing to the discreteness of the analytic observations, the actual analyses resulting from these various combinations of smoothing parameters are different. When derivatives are computed and as stations become more irregularly distributed, these differences increase. An awareness of these potentially significant analysis differences should prompt the analyst to consider carefully the choice of smoothing parameters when applying an objective analysis scheme to real observations.
\end{abstract}

\section{Introduction}

Distance-dependent weighted averaging objective analysis schemes such as those developed by Barnes $(1964,1973)$ and Cressman (1959) have been proven to be extremely useful tools for better understanding atmospheric behavior. Although such schemes no longer

\footnotetext{
${ }^{1}$ This is due in part to two important factors: 1 ) These schemes typically are used to perform univariate analyses and therefore any possible relationships among variables are ignored. 2) The statistical properties of both the background field and the observations are not taken into account.
}

* Current affiliation: Weather Decision Technologies, Inc., Norman, Oklahoma.

+ Additional affiliation: NOAA/National Severe Storms Laboratory, Norman, Oklahoma.

Corresponding author address: Phillip L. Spencer, Weather Decision Technologies, Inc., 1818 W. Lindsey St., D208, Norman, OK, 73069.

E-mail: pspencer@wdtinc.com

DOI: 10.1175/JTECH1995.1

(C) 2007 American Meteorological Society are used to provide the initial conditions to operational numerical weather prediction models, ${ }^{1}$ they still are used frequently for diagnostic purposes. In fact, many recent studies have used various incarnations of the Barnes and Cressman schemes to investigate a diverse range of topics and phenomena, such as snow-to-liquid ratio climatologies (Baxter et al. 2005), a Southern Plains supercell (Dowell et al. 2004), a decaying tropical mesoscale convective system (Geldmeier and Barnes 1997), Florida land breezes (Case et al. 2005), the environment of warm-season elevated thunderstorms (Moore et al. 2003), sea surface temperature modification by hurricanes (Nelson 1998), and decaying extratropical cyclones (Morris and Smith 2001), just to name a few.

Along with its simplicity, one of the primary attractive features of the Barnes analysis scheme is its well-known filtering (response) properties; these properties are established a priori once the analyst chooses the smoothing parameters for a particular application. Therefore, by choosing the smoothing parameters, the analyst controls the scale retention (and removal) characteristics of the analysis scheme. Often the smoothing parameters are chosen such that the 
analysis retains a particular small percentage of the amplitude of the Nyquist wavelength (e.g., Koch et al. 1983).

Perhaps an overlooked aspect of the response function as presented by Barnes $(1964,1973)$ is that it is derived by assuming that the observations are continuous and infinite (unbounded). Meteorological observations are, of course, far from being either continuous or infinite. Because of this, the actual response characteristics of the Barnes analysis scheme differ from the theoretical response characteristics (e.g., Achtemeier 1986; Pauley and Wu 1990; Pauley 1990; Buzzi et al. 1991). The importance of the discreteness of the observations is highlighted by Schlax and Chelton (2002) who assert that "the filtering properties of any smoother can depend as much on the data distribution as on the selection of the smooth[ing] parameters" and by Stephens (1967) who states that "with arbitrary data spacing almost any response may be generated." Therefore, when selecting the smoothing parameters for a particular application of an analysis scheme, the analyst should consider the data distribution characteristics, in addition to the desired scales to resolve and the accuracy and representativeness of the observations.

For a multiple-pass analysis scheme, a desired theoretical amplitude response at a particular wavelength can be derived from an unlimited number of smoothing parameter combinations. The questions that we wish to address are these: Do all combinations of smoothing parameters that would produce equivalent analyses if the observations were continuous and infinite produce equivalent analyses when the observations are discrete and bounded? If not, are the differences significant? We will address these questions - mainly by illustration - by applying an analysis scheme using various "equivalent" combinations of smoothing parameters to analytic observations derived by sampling a one-dimensional sinusoidal wave. We will compare various gridded analyses, including first and second derivatives, using three different measures of analysis error.

In the following sections, we describe how analytic observations are created (section 2) and the Barnes analysis process whereby these observations are mapped onto a grid (section 3). In section 4, we present the results. Finally, section 5 contains a brief summary and concluding remarks.

\section{Creating analytic observations and artificial observing networks}

The error-free scalar observations for this study are derived by sampling an analytic one-dimensional sinu- soidal function. The advantage of using an analytic function is that the scalar field and its spatial derivatives are known at all points within the domain. The $k$ th observation $\left(S_{k}\right)$ is given by the function

$$
S_{k}=\sin \left(\frac{2 \pi}{L} x_{k}\right)
$$

where $L$ is the wavelength and $x_{k}$ is the location of the $k$ th observation.

Artificial observing networks of considerable variety can be obtained simply by sampling the analytic function at different points. For example, a network of 50 regularly distributed observations can be created simply by sampling the analytic function at regular intervals. To create a network of 50 irregularly distributed observations, the analytic function is sampled at irregular intervals. Following the method used by Barnes (1994) and Doswell and Lasher-Trapp (1997), the irregular intervals are determined by randomly displacing each regularly spaced observing location by a distance that is no greater than some fraction of the average data spacing ( $\Delta$; here, $\Delta=1$ arbitrary length unit). This fraction is referred to as the scatter constant (SC) and we choose $\mathrm{SC}$ such that $0.0 \leq \mathrm{SC} \leq 1.0$. By choosing $\mathrm{SC}=0.0$, an observing network is created whose spatial distribution is perfectly regular. In contrast, by choosing $\mathrm{SC}=1.0$, an observing network is created whose spatial distribution is highly irregular, consisting of significant data clustering and large data void regions. $^{2}$

\section{Objective analysis scheme}

Once an analytic observation has been created at each of the 50 stations composing an artificial observing network, the observations are analyzed to a regular grid, whose grid spacing is $0.1 \Delta$. The observations are analyzed to the grid using a two-pass Barnes objective analysis scheme (Barnes 1964, 1973). The weighting function is given by

$$
w_{k}=\exp \left[\frac{-R_{k}^{2}}{\left(c_{i} \Delta\right)^{2}}\right],
$$

where $w_{k}$ is the weight of the $k$ th observation, $R_{k}$ is the distance between a station and a grid point, and $c_{i}$ is the smoothing parameter used during the $i$ th analysis pass. Equation (2) also is used to create "analysis" estimates at stations, except that $R_{k}$ represents the distance be-

\footnotetext{
${ }^{2}$ Doswell and Lasher-Trapp (1997) showed that little or no increase in the irregularity of the station distribution is obtained as SC increases beyond 1.0.
} 
TABLE 1. Various combinations of the smoothing parameters $c_{1}$ and $c_{2}$ used for the three different wavelengths discussed. For all three waves, the values of $c_{1}$ listed in the first columns are used. The values of $c_{2}$ for the $L=5 \Delta, L=10 \Delta$, and $L=15 \Delta$ waves are shown in columns 2,3 , and 4 , respectively. The theoretical amplitude response is 0.78 for each combination of the smoothing parameters listed for the $L=5 \Delta$ wave, 0.98 for each combination listed for the $L=10 \Delta$ wave, and 0.996 for each combination listed for the $L=15 \Delta$ wave.

\begin{tabular}{cccc}
\hline \hline & $\begin{array}{c}R(L=5 \Delta) \\
=0.78\end{array}$ & $\begin{array}{c}R(L=10 \Delta) \\
=0.98\end{array}$ & $\begin{array}{c}R(L=15 \Delta) \\
=0.996\end{array}$ \\
\cline { 2 - 4 }$c_{1}$ & $c_{2}$ & $c_{2}$ & $c_{2}$ \\
\hline 1.3 & 1.23 & 1.19 & 1.15 \\
1.5 & 1.09 & 1.04 & 1.00 \\
1.7 & 0.99 & 0.92 & 0.88 \\
1.9 & 0.93 & 0.84 & 0.79 \\
2.1 & 0.89 & 0.77 & 0.72 \\
2.3 & 0.86 & 0.71 & 0.67 \\
\hline
\end{tabular}

tween observations. These estimates are used during the second (correction) pass. ${ }^{3}$

Barnes (1973) and Koch et al. (1983) have shown that the theoretical amplitude response $(R)$ after the application of a two-pass Barnes scheme is

$$
\begin{aligned}
R\left(c_{1}, c_{2}, N\right)= & \exp \left[-\left(\frac{c_{1} \pi}{2 N}\right)^{2}\right] \\
& +\left\{1-\exp \left[-\left(\frac{c_{1} \pi}{2 N}\right)^{2}\right]\right\} \\
& \times \exp \left[-\left(\frac{c_{2} \pi}{2 N}\right)^{2}\right],
\end{aligned}
$$

where $c_{1}$ is the smoothing parameter used during the first pass of the analysis scheme, $c_{2}$ is the smoothing parameter used during the second pass, and $N$ is the nondimensional Nyquist multiple $[L /(2 \Delta)]$. Any number of combinations of $c_{1}$ and $c_{2}$ can be used to produce an analysis whose theoretical amplitude response at some Nyquist multiple is some desired value. For example, combinations of $c_{1}$ and $c_{2}$ producing theoretical amplitude responses of $0.78,0.98$, and 0.996 for $L=5 \Delta$, $L=10 \Delta$, and $L=15 \Delta$ waves, respectively, are shown in Table 1. We emphasize that these are theoretical amplitude responses, which are valid only for data that are continuous and infinite (unbounded). In the real world, of course, observations are discrete-generally collected at irregular spatial intervals-and bounded. Therefore, the actual response differs from the theoret-

\footnotetext{
${ }^{3}$ Bilinear interpolation is a popular alternative for creating these "analysis" estimates at stations.
}

ical response in meteorological applications. In the following section, we will illustrate how different combinations of smoothing parameters that provide equivalent theoretical responses (analyses) in fact produce different actual responses (analyses).

\section{Results}

\section{a. Measures of analysis error}

Three metrics are used to evaluate the analyses. First, the root-mean-square error (rmse) is computed according to

$$
\text { rmse }=\sqrt{\frac{\sum_{i}\left(S_{g}-S_{a}\right)^{2}}{N_{g}}},
$$

where $S_{g}$ represents an analysis value (zeroth, first, or second derivative), $S_{a}$ represents the corresponding analytic (true) value, ${ }^{4}$ and $N_{g}$ is the number of grid points within the verification domain.

The two other measures of analysis error are the amplitude response and phase shift. Ideally, the actual amplitude response at each grid point is equivalent to the theoretical value implied by the response function [Eq. (3)]. Since the observations are discrete and bounded, this will not occur (Pauley and $\mathrm{Wu}$ 1990; Buzzi et al. 1991). The deviation of the actual amplitude response from the theoretical value can provide a measure of analysis error. Also, ideally, the phase shift of the gridded observations is zero after the application of this type of analysis scheme. The actual amplitude responses and phase shifts are computed according to the method described by Askelson and Straka (2005) and Askelson et al. (2005), wherein equivalent one-pass weights are required. ${ }^{5}$ These equivalent one-pass weights are computed following the approach of Caracena (1987) and Schlax and Chelton (2002).

For the Barnes scheme, analysis errors associated with lateral boundaries intrude toward the interior of the analysis domain by a distance related to the value of

\footnotetext{
${ }^{4}$ Analytic values of the zeroth derivative are obtained by replacing $x_{k}$ in Eq. (1) with the locations of the grid points. Similarly, analytic values of the first and second derivatives are obtained by taking the appropriate derivatives of Eq. (1) and replacing $x_{k}$ with the locations of the grid points.

${ }^{5}$ The equivalent one-pass weights are the weights that, when applied to the observations using a one-pass analysis scheme, produce the same analysis as that produced using a multiple-pass scheme.
} 

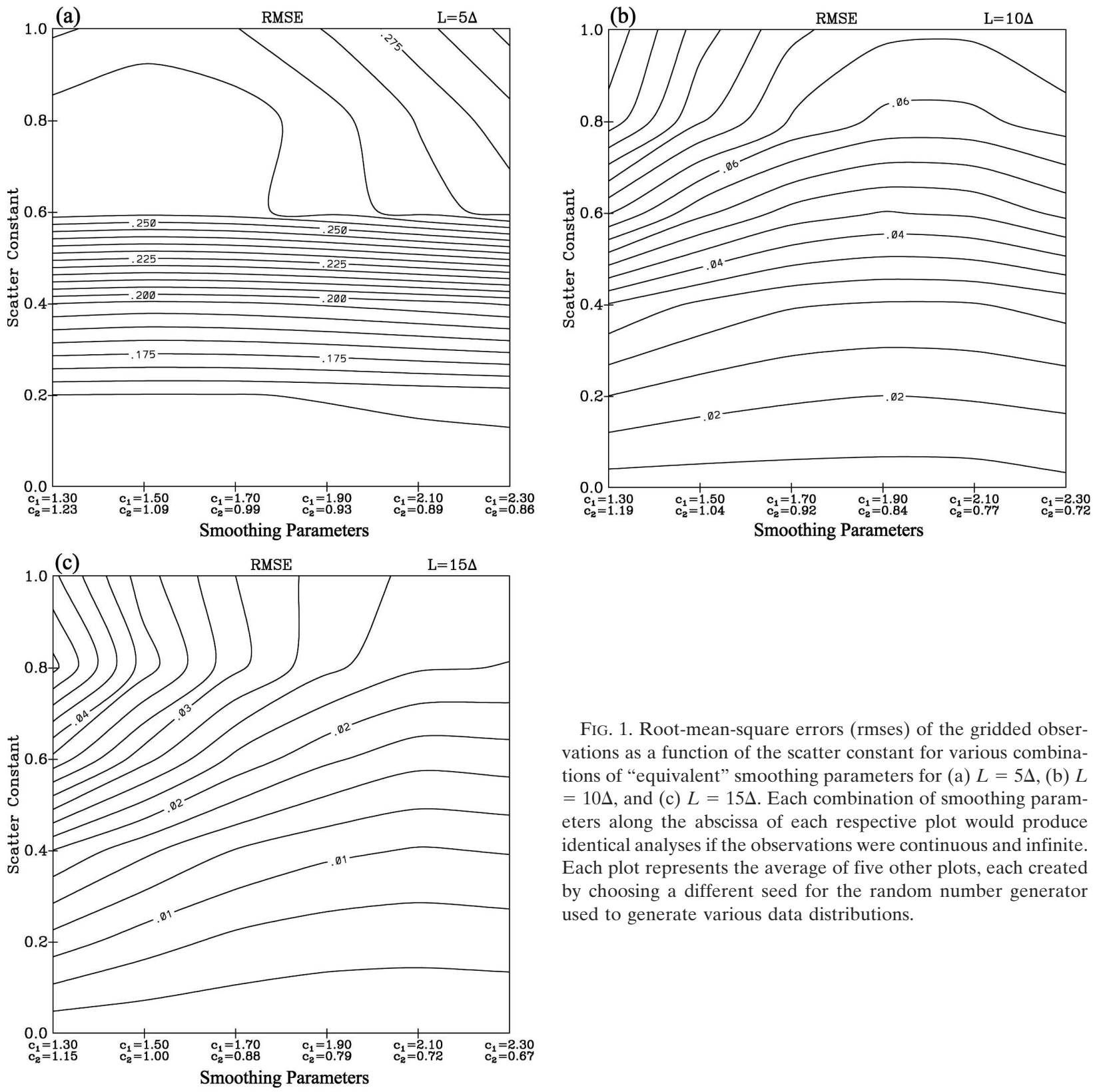

FIG. 1. Root-mean-square errors (rmses) of the gridded observations as a function of the scatter constant for various combinations of "equivalent" smoothing parameters for (a) $L=5 \Delta$, (b) $L$ $=10 \Delta$, and (c) $L=15 \Delta$. Each combination of smoothing parameters along the abscissa of each respective plot would produce identical analyses if the observations were continuous and infinite. Each plot represents the average of five other plots, each created by choosing a different seed for the random number generator used to generate various data distributions.

the smoothing parameter (e.g., Caracena et al. 1984; Doswell and Lasher-Trapp 1997). We avoid contamination of the error statistics associated with the two lateral boundaries by limiting the verification domain to the innermost one-half of the analysis domain.

\section{b. Broad overview}

To see how different combinations of the smoothing parameters $c_{1}$ and $c_{2}$ produce different analyses for three different wavelengths $(L=5 \Delta, L=10 \Delta$, and $L=$

$15 \Delta$ ) and six different values of SC, consider Figs. $1-5{ }^{6}$ Clearly, each of these figures suggests that as the irregularity of the station distribution increases (i.e., as

${ }^{6}$ As indicated by Eq. (4), the actual analytic function and its derivatives are used to compute the rmses that are shown in Figs. $1-3$. We note that if $S_{a}$ is redefined to be the analytic function and its derivatives damped according to the desired amplitude response, the patterns of the contours remain almost unchanged, although the magnitudes of the errors decrease somewhat. Therefore, however $S_{a}$ is defined, the conclusions presented herein are consistent. 

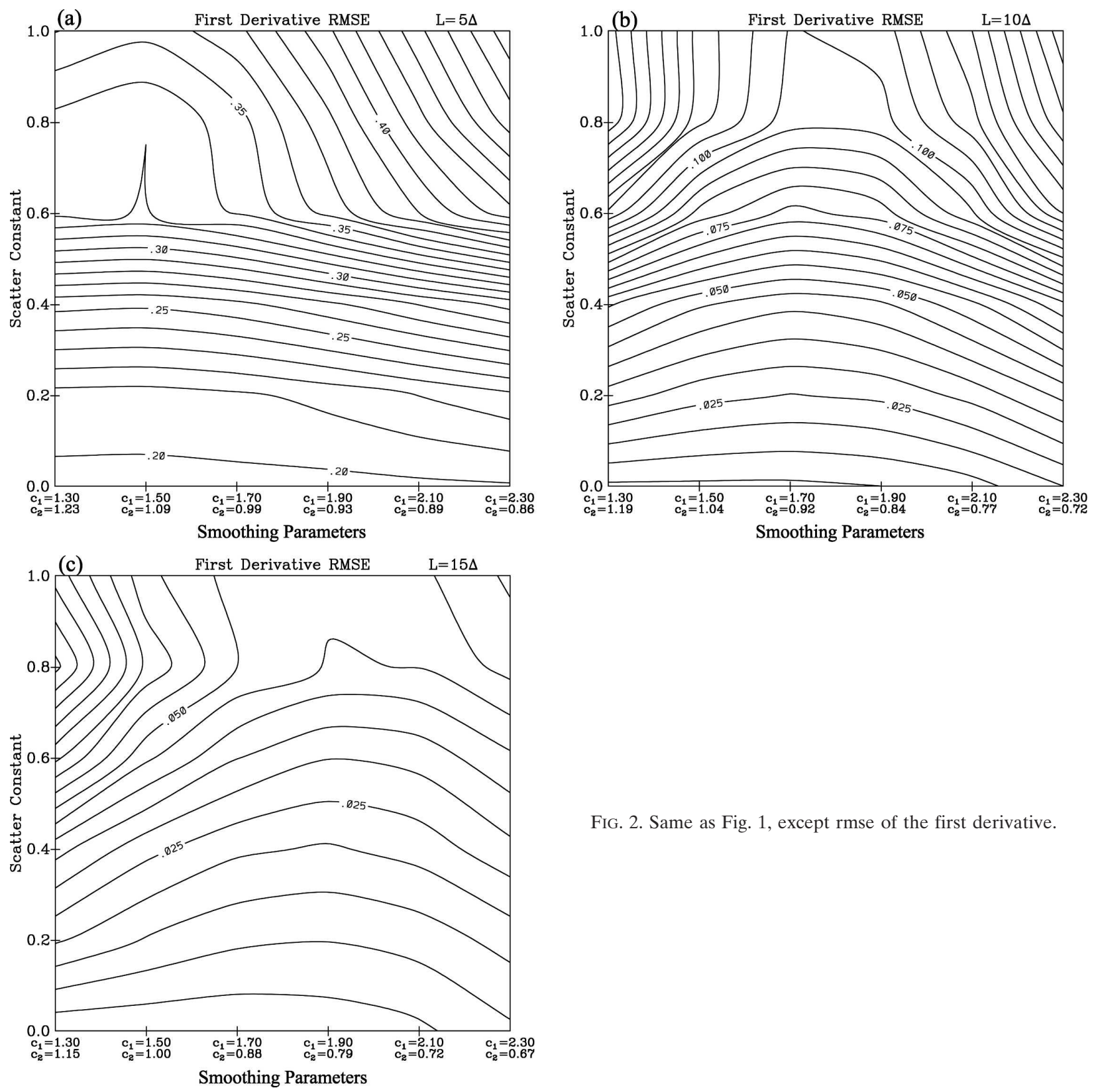

FIG. 2. Same as Fig. 1, except rmse of the first derivative.

SC increases), the analysis error increases. This result is consistent with the findings of Smith et al. (1986), Barnes (1994), Doswell and Lasher-Trapp (1997), and Spencer and Gao (2004). Also, the errors tend to decrease as the wavelength increases. What is of most interest in this study, however, is how the analysis errors vary as a function of different combinations of the smoothing parameters. For example, Fig. 1 suggests that carefully selecting the smoothing parameters is most important for highly irregularly distributed observations (e.g., $\mathrm{SC}>0.6$ ); that is, the most significant variability in the rmse for different combinations of

the smoothing parameters occurs for the data networks whose stations are highly irregularly distributed. For observation networks whose stations are somewhat regularly spaced, any number of different smoothing parameter combinations results in more or less equivalent analyses. For the $L=10 \Delta$ wave (Fig. 1b), the smoothing parameter combination that best minimizes the scalar rmse is one that contains a relatively high (low) value of $c_{1}\left(c_{2}\right)$. For the $L=15 \Delta$ wave (Fig. 1c), the desired value of $c_{1}\left(c_{2}\right)$ is even larger (smaller).

When the derivative analyses are considered (Figs. 2 

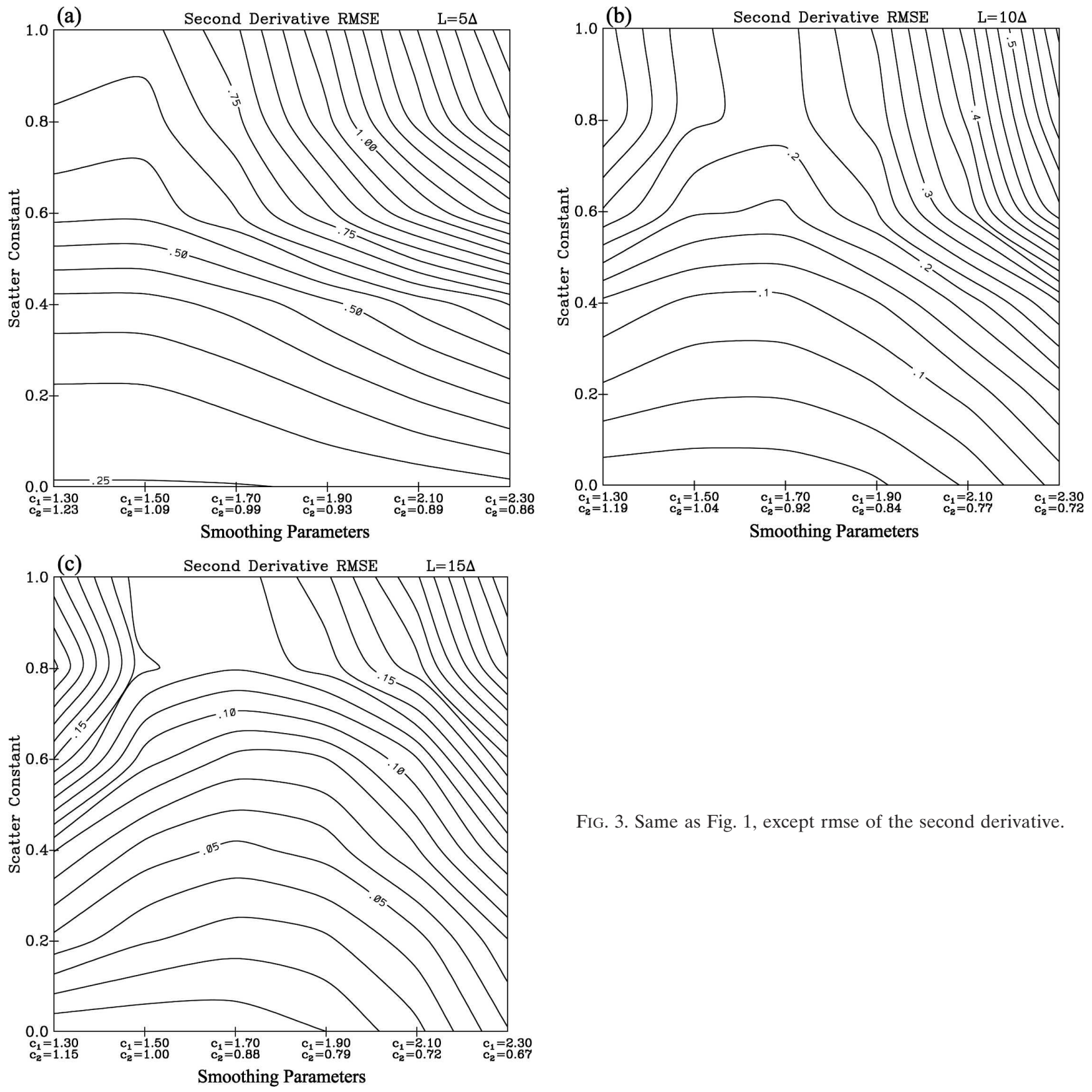

FIG. 3. Same as Fig. 1, except rmse of the second derivative.

and 3), ${ }^{7}$ it becomes even more obvious that careful consideration should be taken when choosing the smoothing parameters, especially for a network of stations whose distribution is irregular. For example, for $L=$ $10 \Delta$ and $\mathrm{SC}=0.8$, the rmse of the first derivative computed from an analysis created by choosing $c_{1}=1.3$ and $c_{2}=1.19$ is nearly twice the rmse computed from an analysis created by choosing $c_{1}=1.9$ and $c_{2}=0.84$ (Fig.

\footnotetext{
${ }^{7}$ The spatial derivatives are computed using a second-order finite-differencing scheme applied to the scalar analysis.
}

2b). Also, as before, it is clear that there are distinct combinations of smoothing parameters that minimize the rmse for a given scatter constant (Figs. 2 and 3). Interestingly, the combination of smoothing parameters that minimizes the rmses of the gridded observations is not the same combination that minimizes the rmses of the first derivative, which, in turn, is different still from the combination that minimizes the rmses of the second derivative. In fact, with each successive derivative, the value of $c_{1}\left(c_{2}\right)$ that minimizes the rmses decreases (increases). This may be an important factor to consider, depending on the goal of the analysis. 

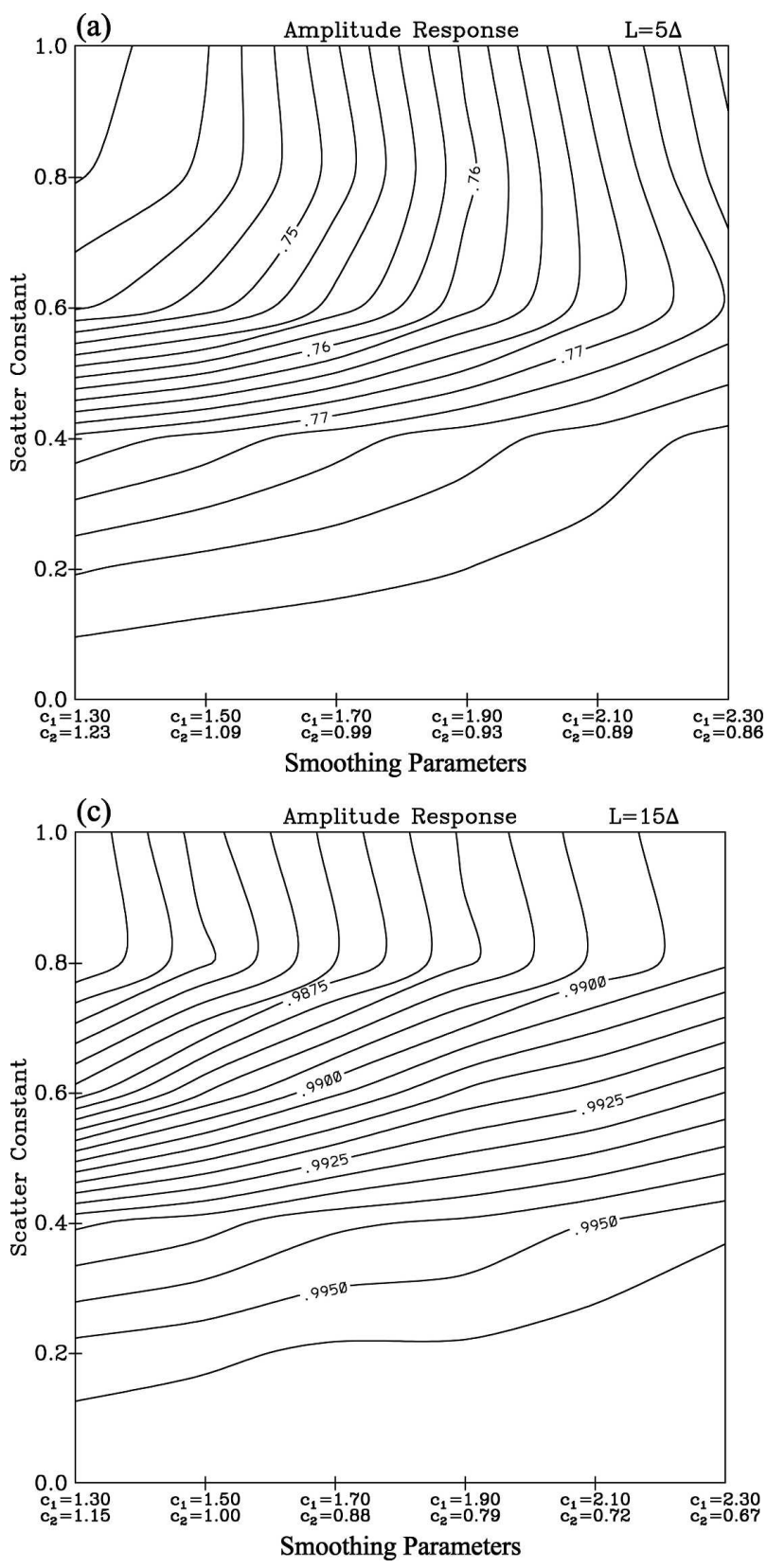

Even for regularly distributed observations $(\mathrm{SC}=$ 0.0 ), there are differences in the derivative analysesespecially the second derivative-at long wavelengths for different combinations of the smoothing parameters (Figs. 3b and 3c). In fact, for such distributions, the rmse of the second derivative can vary by a factor of nearly 5 for the larger two waves, depending on the choice of the smoothing parameters.

The amplitude responses for the three different waves (Fig. 4) generally give results similar to those found by examining the rmses. Specifically, 1) the analysis error tends to increase as the irregularity of the

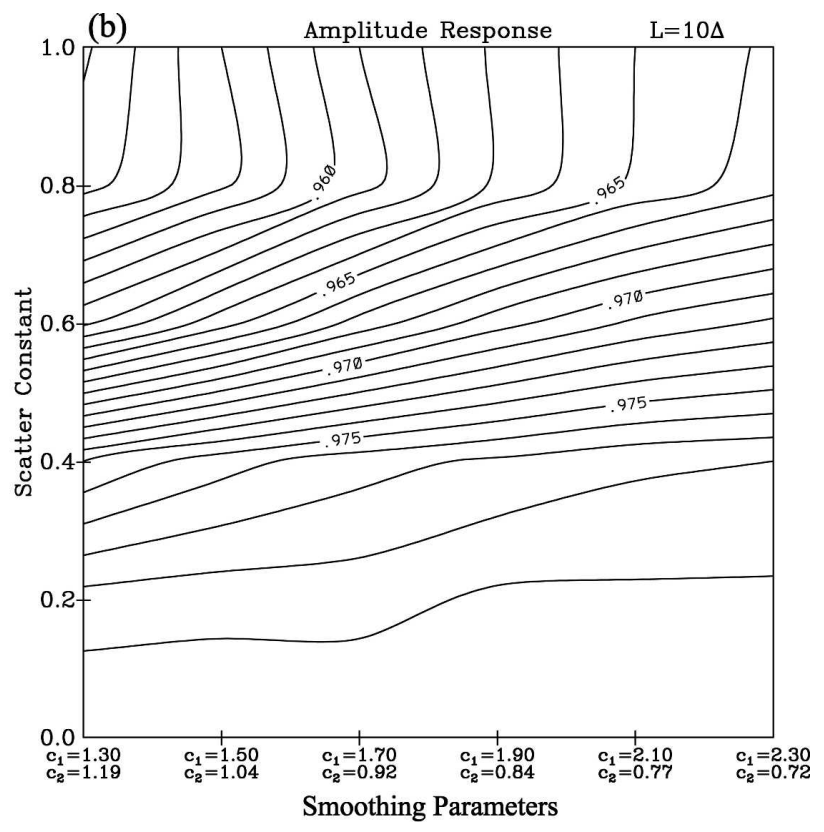

FIG. 4. Same as Fig. 1, except amplitude response averaged across the verification domain. The theoretical amplitude response is 0.78 for (a), 0.98 for (b), and 0.996 for (c).

station distribution increases and 2) the largest variability in the quality of the analyses for different combinations of the smoothing parameters occurs for highly irregularly distributed observations. Unlike the rmses, however, the combination of smoothing parameters that best replicates the theoretical amplitude response generally is the one with the highest (lowest) value of $c_{1}$ $\left(c_{2}\right)$ that was considered in this study. However, when the phase shift is considered (Fig. 5), the best combination of smoothing parameters - at least for the $L=$ $10 \Delta$ and $L=15 \Delta$ waves-generally is one with a slightly lower (higher) value of $c_{1}\left(c_{2}\right)$. As before, the 

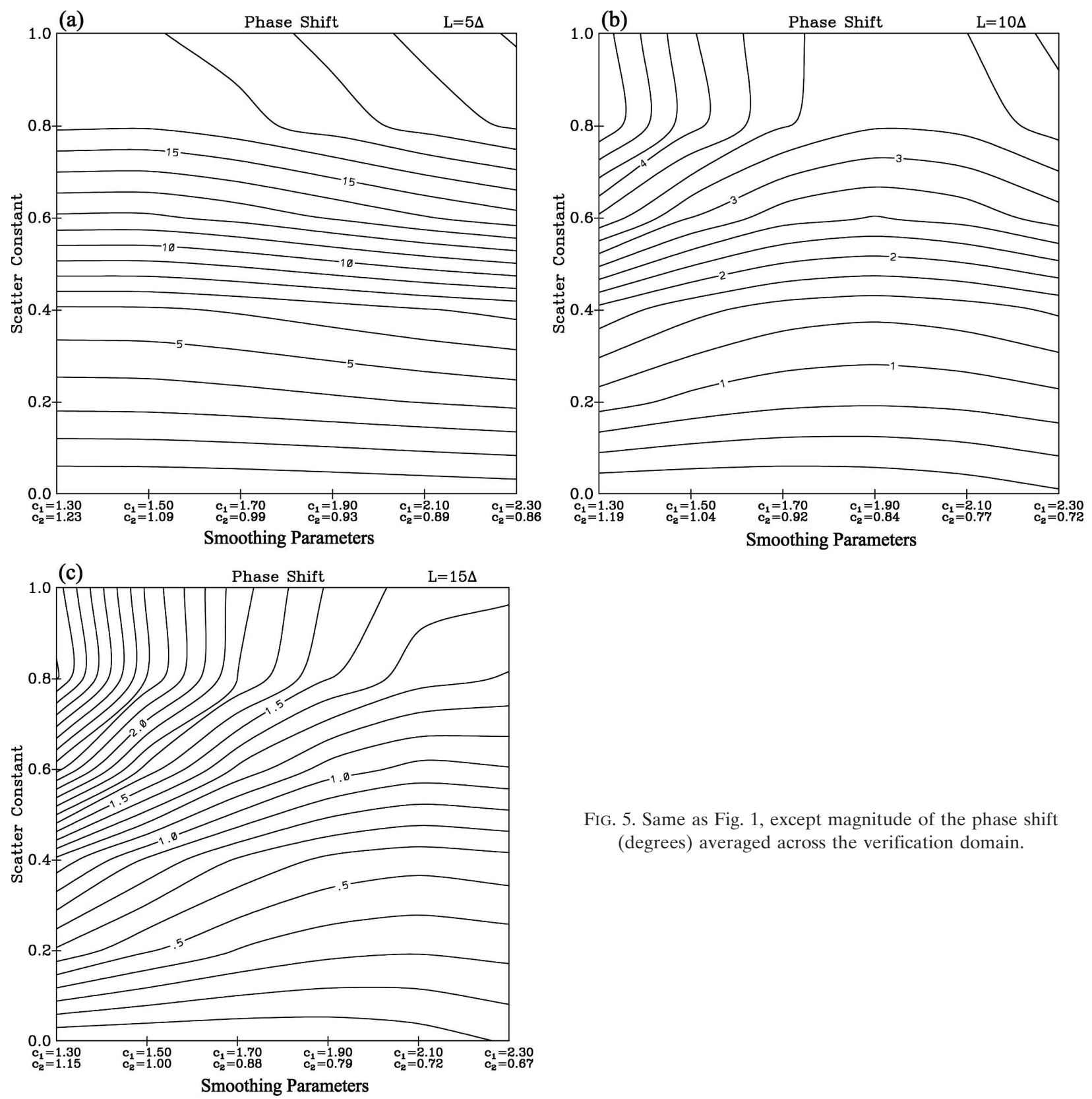

FIG. 5. Same as Fig. 1, except magnitude of the phase shift (degrees) averaged across the verification domain.

greatest variability in the quality of the analyses for various combinations of the smoothing parameters occurs for the data networks composed of highly irregularly distributed observations. For regularly distributed observations, the phase shifts are negligible for all combinations of the smoothing parameters and for all three wavelengths considered. Not surprisingly, the most significant phase shifts occur for the smallest wave considered $(L=5 \Delta)$, the one that is most poorly sampled. As the wavelength increases and the wave becomes increasingly better sampled, the phase shifts decrease.

\section{c. Specific examples}

Two analyses for a particular data distribution created by choosing $\mathrm{SC}=0.8$ for three different wavelengths are shown as dashed curves in Figs. 6-8. Clearly, the discreteness of the observations (indicated by the small asterisks along the abscissa) is treated differently by the two different combinations of smoothing parameters, thereby producing distinct analyses for the $L=5 \Delta$ wave (Fig. 6a). As shown in the previous section, derivatives tend to magnify the differences in 

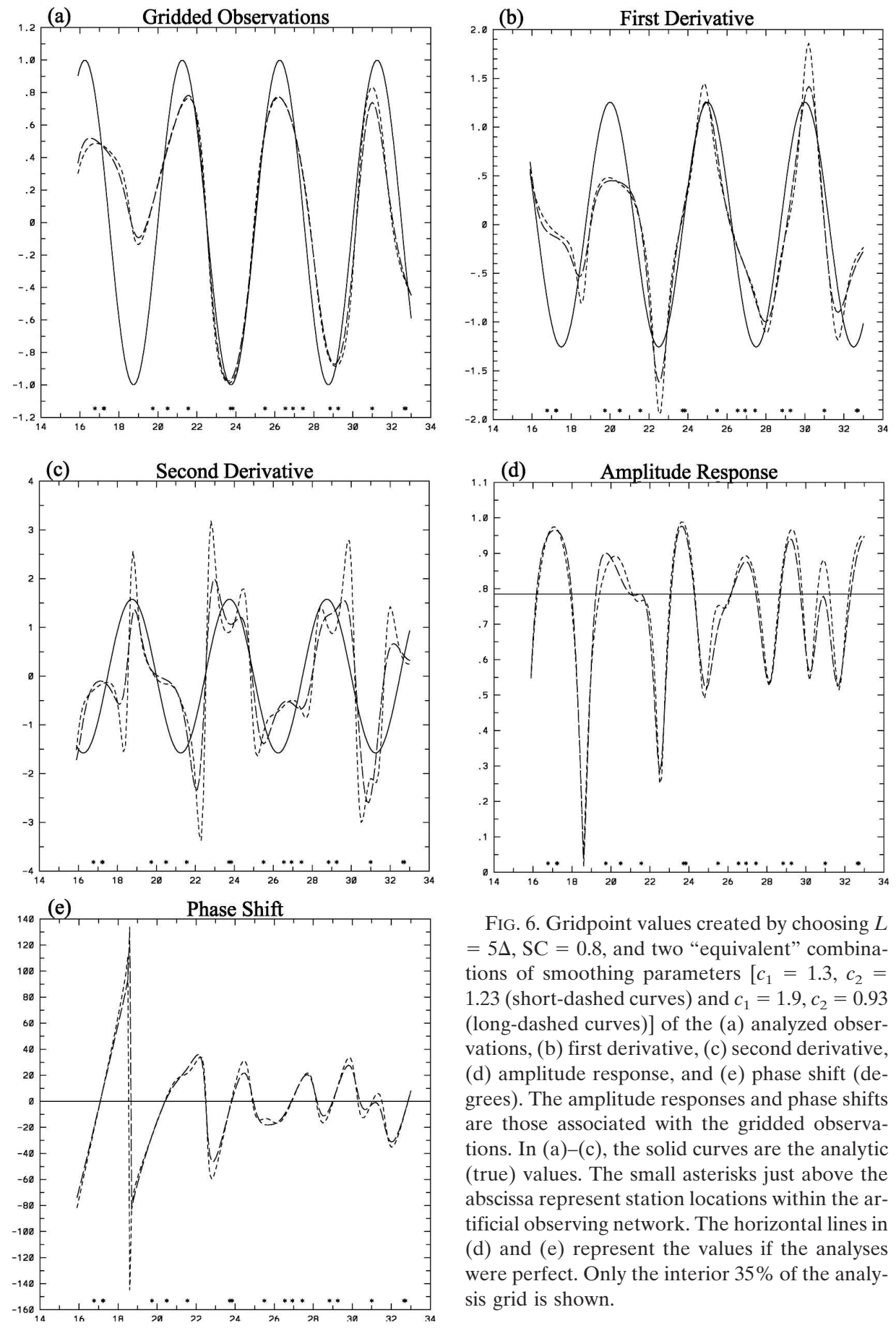

FIG. 6. Gridpoint values created by choosing $L$ $=5 \Delta, \mathrm{SC}=0.8$, and two "equivalent" combinations of smoothing parameters $\left[c_{1}=1.3, c_{2}=\right.$ 1.23 (short-dashed curves) and $c_{1}=1.9, c_{2}=0.93$ (long-dashed curves)] of the (a) analyzed observations, (b) first derivative, (c) second derivative, (d) amplitude response, and (e) phase shift (degrees). The amplitude responses and phase shifts are those associated with the gridded observations. In (a)-(c), the solid curves are the analytic (true) values. The small asterisks just above the abscissa represent station locations within the artificial observing network. The horizontal lines in (d) and (e) represent the values if the analyses were perfect. Only the interior $35 \%$ of the analysis grid is shown.

the analyses (Figs. 6b and 6c). As expected, the poorest portions of the analyses are within significant data voids. For example, at $x=18.5$, the analyses are not able to capture the large dip in the analytic wave owing to inadequate sampling in this region (Fig. 6a). The poorest portions of the analyses also are where the most significant differences between the analyses tend

to occur. Figures $6 \mathrm{~d}$ and 6e clearly illustrate how the analysis error (here expressed in terms of amplitude response and phase shift) can vary greatly across the analysis domain. At only about 13 of the 171 grid points within the interior portion of the domain does the actual amplitude response equal the theoretical value of 0.78. At the other grid points, the actual amplitude re- 

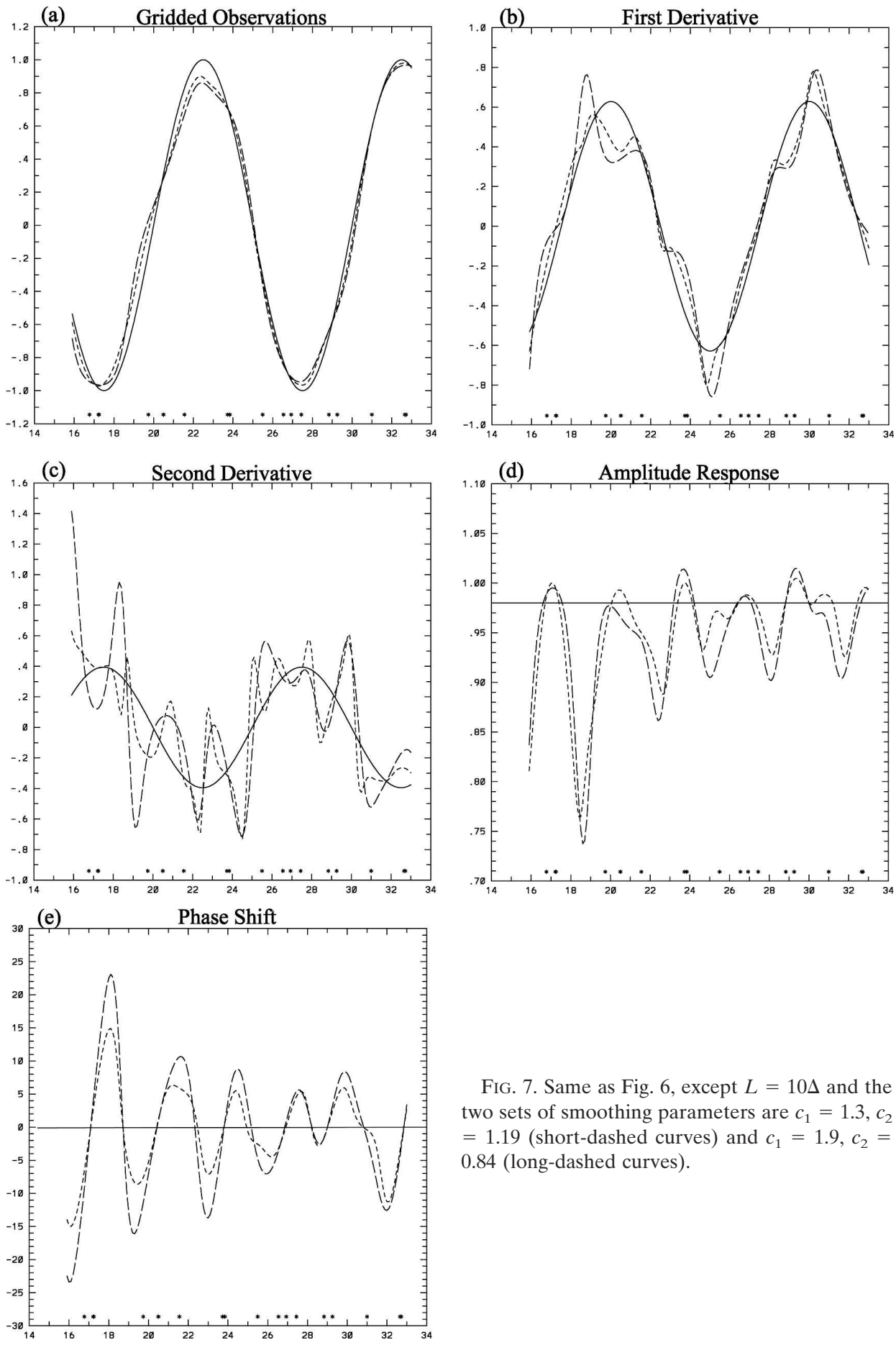

FIG. 7. Same as Fig. 6, except $L=10 \Delta$ and the two sets of smoothing parameters are $c_{1}=1.3, c_{2}$ $=1.19$ (short-dashed curves) and $c_{1}=1.9, c_{2}=$ 0.84 (long-dashed curves).

sponse exceeds the theoretical value by as much as $25 \%$ and is below the theoretical value by as much as nearly $100 \%$. Similarly, the same number of grid points (a mere $8 \%$ ) have the desired phase shift of zero degrees.

As the wavelength increases to $L=10 \Delta$, nontrivial differences in the analyses remain apparent (Figs. $7 \mathrm{a}-\mathrm{c}$ ), even though the wave is better sampled (relative to the wavelength) than the $L=5 \Delta$ wave. As before, the discreteness of the observations forces the analyses from the two smoothing parameter combinations to differ, although the differences generally 

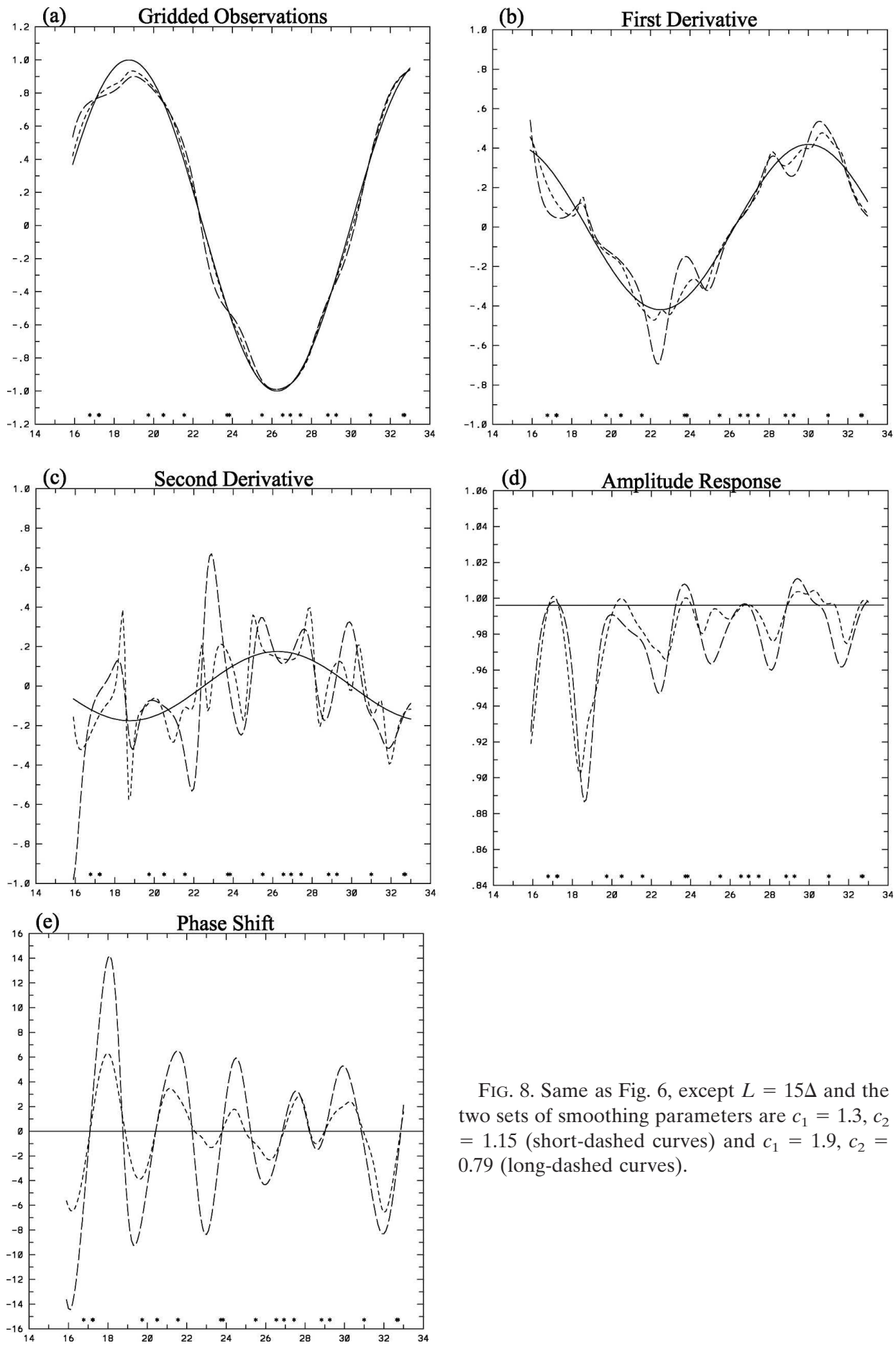

FIG. 8. Same as Fig. 6, except $L=15 \Delta$ and the two sets of smoothing parameters are $c_{1}=1.3, c_{2}$ $=1.15$ (short-dashed curves) and $c_{1}=1.9, c_{2}=$ 0.79 (long-dashed curves).

are less than for the $L=5 \Delta$ wave. Indeed, the amplitude responses and phase shifts for the $L=10 \Delta$ analyses are closer to the ideal values than they are for the $L=5 \Delta$ analyses.

Finally, for the $L=15 \Delta$ wave (Fig. 8), differences between the two analyses remain evident because of the discreteness of the observations, even though this is a well-sampled wave. However, because this is such a well sampled wave, the differences in the analyses (and the actual analyses errors themselves) are relatively small. As before, large data gaps tend to degrade the analyses in those regions. 


\section{Summary and discussion}

In this study, we have demonstrated the importance of carefully choosing the smoothing parameters within a multiple-pass Barnes objective analysis scheme. An infinite number of smoothing parameter combinations would produce equivalent analyses if the data were continuous and infinite. However, because real observations are discrete and contained within bounded domains, these same combinations of the smoothing parameters actually produce different analyses. The differences become increasingly apparent as spatial derivatives of increasing order are computed, an important fact considering the prevalence of spatial derivatives within many of the important prognostic and diagnostic equations in meteorology. We also found that the differences between analyses created by different "equivalent" combinations of smoothing parameters are most apparent for observational networks whose stations are highly irregularly distributed. Differences between the analyses-and, in fact, analyses errors themselves-tend to be relatively small when the observations are regularly distributed.

Owing to our use of errorless, analytic observations and hypothetical observing networks, we cannot make definitive statements regarding the "correct" choice of smoothing parameters for a particular application. However, we do offer the following broad guidelines for minimizing analysis errors when applying a two-pass scheme: 1) Use a relatively large first-pass smoothing parameter $\left(c_{1}\right)$. This conclusion is consistent with Barnes (1994) who stated that "if the first-pass analysis contains spurious short wavelengths whose amplitudes are significant, second- and higher-pass analyses will exhibit those same spurious details even more incorrectly." The smoothing parameter must not be too large, however, or else boundary errors will be drawn well within the interior of the analysis domain (Achtemeier 1986). 2) Reduce the first-pass smoothing parameter $\left(c_{1}\right)$ and increase the second-pass smoothing parameter $\left(c_{2}\right)$ as spatial derivatives of increasing order are desired. Our experiments indicate that there is no single optimum combination of smoothing parameters with respect to the fields and their spatial derivatives. 3) Superimpose analytic data onto the specific observational network of interest in order to determine the best combination(s) of smoothing parameters. ${ }^{8}$

Although we have investigated the properties of the Barnes scheme only, we recognize that the Cressman scheme (1959) remains a popular alternative for dis-

\footnotetext{
${ }^{8}$ This is a suggestion also given by Barnes (1994).
}

tance-dependent weighted averaging. An exhaustive comparison of the Barnes and Cressman schemes is beyond the desired scope of this work, but we do wish to point out that even single-pass versions of these two schemes configured to produce identical analyses for continuous and infinite observations in fact produce distinguishable analyses when the observations are discrete and bounded (e.g., Fig. 9). [This is because the properties of the observation distribution result in different amplitude modulations and phase shifts (not shown) for the two analysis schemes despite their "equivalent" design.] Therefore, we conclude that not only is the choice of the smoothing parameters within a multiple-pass objective analysis scheme an important consideration for the analyst, but the choice of the form of the weighting function itself (Barnes versus Cressman) should be considered, as well.

When systematic differences in data density are encountered such as with radar observations, the smoothing parameter can be chosen to be a function of data density (e.g., Trapp and Doswell 2000; Askelson et al. 2000). While providing the advantage of retaining more detail where justified by the data, such inhomogeneous schemes have the disadvantage of producing variable local spectra as a result of variations in data density. This can be especially troublesome for diagnosis of moving weather systems, when analysis changes due to data density variations can become convolved with actual time tendencies in the field, as discussed by Trapp and Doswell (2000). Statistical analysis techniques that take into account the distribution of the observations as well as their error characteristics also can provide a useful alternative that has some advantages (and also some disadvantages) compared to simpler Barnes-type schemes. One such example of a statistical objective analysis scheme is that of Bratseth (1986), which is a successive correction approach that converges toward a limit that is optimal in a narrowly defined statistical sense.

Finally, the derivatives in this study were computed by applying a finite differencing scheme to the gridded observations. Although this remains a popular method for estimating spatial derivatives, abundant evidence exists to argue that a gridded field of spatial derivatives is best computed by applying an analysis scheme to derivative estimates that are computed directly from the observations (e.g., Schaefer and Doswell 1979; Doswell and Caracena 1988; Spencer and Doswell 2001). In the future, we hope to compare these two techniques for derivative estimation further by explaining their differences in terms of amplitude and phase errors. 
(a)

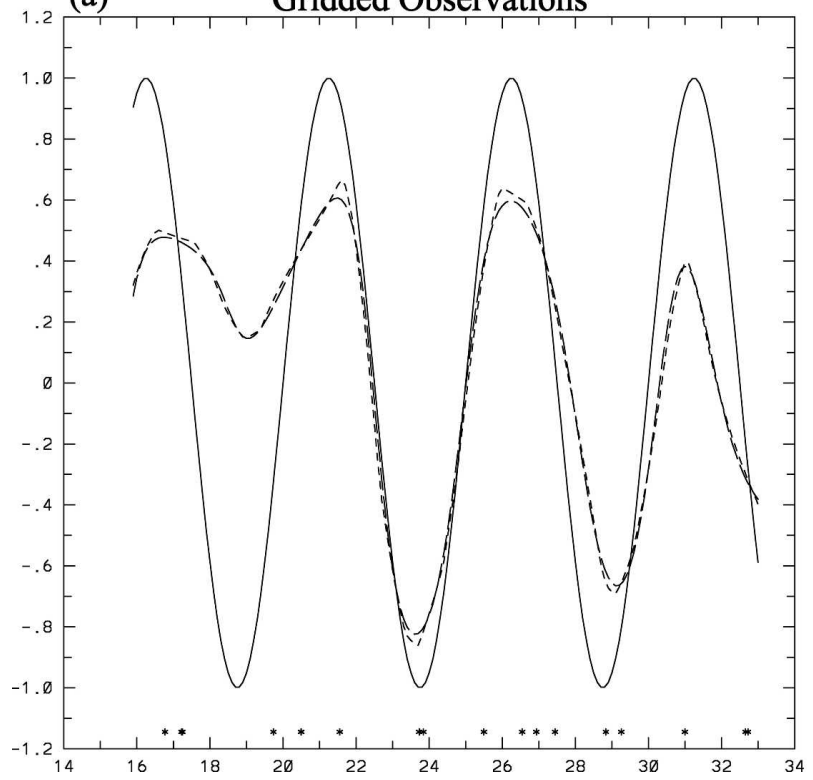

(b)

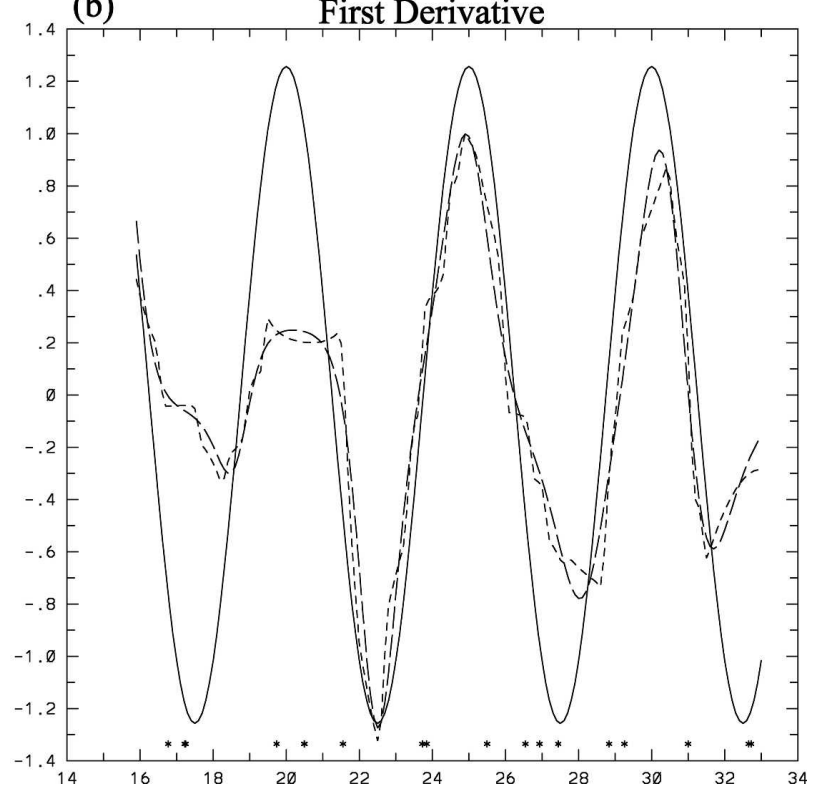

FIG. 9. Gridpoint values created by choosing $L=5 \Delta$ and $\mathrm{SC}=0.8$ for "equivalent" one-pass configurations of the Barnes (long-dashed curves) and Cressman (short-dashed curves) schemes of the (a) analyzed observations and (b) first derivative. The smoothing parameter for the Barnes analysis is $c_{1}=1.3$ and the radius of influence (ROI) for the Cressman analysis is ROI $=2.1572$. Each of these choices produces an amplitude response of 0.51 at $L=5 \Delta$ for continuous and unbounded observations. The solid curves are the analytic (true) values. The small asterisks just above the abscissa represent station locations within the artificial observing network. Only the interior $35 \%$ of the analysis grid is shown.

Acknowledgments. We thank Dr. David Stensrud, Mr. Mike Splitt, and an anonymous reviewer for providing thoughtful comments that helped us improve our manuscript. Major funding for this research was provided under NOAA-OU Cooperative Agreement NA17RJ1227. Partial funding for the second author was provided by the Army High Performance Computing Research Center (AHPCRC) under the auspices of the Department of the Army, Army Research Laboratory (ARL), under Cooperative Agreement DAAD1901-2-0014. The content does not necessarily reflect the position or the policy of the government and no official endorsement should be inferred.

\section{REFERENCES}

Achtemeier, G. L., 1986: The impact of data boundaries upon a successive corrections objective analysis of limited-area datasets. Mon. Wea. Rev., 114, 40-49.

Askelson, M. A., and J. M. Straka, 2005: Response functions for arbitrary weight functions and data distributions. Part I: Framework for interpreting the response function. Mon. Wea. Rev., 133, 2117-2131.

_ - J. Aubagnac, and J. M. Straka, 2000: An adaptation of the Barnes filter applied to the objective analysis of radar data. Mon. Wea. Rev., 128, 3050-3082.

_ - P. M. Pauley, and J. M. Straka, 2005: Response functions for arbitrary weight functions and data distributions. Part II: Re- sponse function derivation and verification. Mon. Wea. Rev., 133, 2132-2147.

Barnes, S. L., 1964: A technique for maximizing details in numerical weather map analysis. J. Appl. Meteor., 3, 396-409.

, 1973: Mesoscale objective analysis using weighted timeseries observations. NOAA Tech. Memo. ERL NSSL-62, National Severe Storms Laboratory, Norman, OK, 41 pp. [NTIS COM-73-10781.]

_ 1994: Applications of the Barnes objective analysis scheme. Part I: Effects of undersampling, wave position, and station randomness. J. Atmos. Oceanic Technol., 11, 1433-1448.

Baxter, M. A., C. E. Graves, and J. T. Moore, 2005: A climatology of snow-to-liquid ratio for the contiguous United States. Wea. Forecasting, 20, 729-744.

Bratseth, A. M., 1986: Statistical interpolation by means of successive corrections. Tellus, 38A, 439-447.

Buzzi, A., D. Gomis, M. A. Pedder, and S. Alonso, 1991: A method to reduce the adverse impact that inhomogeneous station distributions have on spatial interpolation. Mon. Wea. Rev., 119, 2465-2491.

Caracena, F., 1987: Analytic approximation of discrete field samples with weighted sums and the gridless computation of field derivatives. J. Atmos. Sci., 44, 3753-3768.

_, S. L. Barnes, and C. A. Doswell III, 1984: Weighting function parameters for objective interpolation of meteorological data. Proc. 10th Conf. on Weather Forecasting and Analysis, Clearwater Beach, FL, Amer. Meteor. Soc., 109-116.

Case, J. L., M. M. Wheeler, J. Manobianco, J. W. Weems, and W. P. Roeder, 2005: A 7-yr climatological study of land breezes over the Florida spaceport. J. Appl. Meteor., 44, 340356. 
Cressman, G., 1959: An operational objective analysis system. Mon. Wea. Rev., 87, 367-374.

Doswell, C. A., III, and F. Caracena, 1988: Derivative estimation from marginally sampled vector point functions. J. Atmos. Sci., 45, 242-253.

— irregularity in an observing network. J. Atmos. Oceanic Technol., 14, 120-132.

Dowell, D. C., F. Zhang, L. J. Wicker, C. Snyder, and N. A. Crook, 2004: Wind and temperature retrievals in the 17 May 1981 Arcadia, Oklahoma, supercell: Ensemble Kalman filter experiments. Mon. Wea. Rev., 132, 1982-2005.

Geldmeier, M. F., and G. M. Barnes, 1997: The "footprint" under a decaying tropical mesoscale convective system. Mon. Wea. Rev., 125, 2879-2895.

Koch, S. E., M. desJardins, and P. J. Kocin, 1983: An interactive Barnes objective map analysis scheme for use with satellite and conventional data. J. Climate Appl. Meteor., 22, 14871503.

Moore, J. T., F. H. Glass, C. E. Graves, S. M. Rochette, and M. J. Singer, 2003: The environment of warm-season elevated thunderstorms associated with heavy rainfall over the central United States. Wea. Forecasting, 18, 861-878.

Morris, W. E., Jr., and P. J. Smith, 2001: Cyclolysis: A diagnosis of two extratropical cyclones. Mon. Wea. Rev., 129, 2714-2729.

Nelson, N. B., 1998: Spatial and temporal extent of sea surface temperature modifications by hurricanes in the Sargasso Sea during the 1995 season. Mon. Wea. Rev., 126, 1364-1368.

Pauley, P. M., 1990: On the evaluation of boundary errors in the Barnes objective analysis scheme. Mon. Wea. Rev., 118, 12031210.

- and X. Wu, 1990: The theoretical, discrete, and actual response of the Barnes objective analysis scheme for one- and two-dimensional fields. Mon. Wea. Rev., 118, 1145-1163.

Schaefer, J. T., and C. A. Doswell III, 1979: On the interpolation of a vector field. Mon. Wea. Rev., 107, 458-476.

Schlax, M. G., and D. B. Chelton, 2002: Filter transfer functions for the method of successive corrections. Mon. Wea. Rev., 130, 372-385.

Smith, D. R., M. E. Pumphry, and J. T. Snow, 1986: A comparison of errors in objectively analyzed fields for uniform and nonuniform station distributions. J. Atmos. Oceanic Technol., 3, 84-97.

Spencer, P. L., and C. A. Doswell III, 2001: A quantitative comparison between traditional and line integral methods of derivative estimation. Mon. Wea. Rev., 129, 2538-2554.

_ , and J. Gao, 2004: Can gradient information be used to improve variational objective analysis? Mon. Wea. Rev., 132, 2977-2994.

Stephens, J. J., 1967: Filtering responses of selected distantdependent weight functions. Mon. Wea. Rev., 95, 45-46.

Trapp, R. J., and C. A. Doswell III, 2000: Radar data objective analysis. J. Atmos. Oceanic Technol., 17, 105-120. 